\title{
Distance in Degree Splitting Graphs
}

\author{
*Selvam Avadayappan, M. Bhuvaneshwari and Rajeev Gandhi \\ Research Department of Mathematics, VHNSN College, Virudhunagar - 626001, India. \\ Corresponding Author: Selvam Avadayappan
}

\begin{abstract}
For a graph $G(V, E)$, if $V_{i}$ denote the set of all vertices of degree $i$, the degree splitting graph $D S(G)$ is the graph obtained from $G$ by adding new vertices $w_{i}$ for each $V_{i}$ with $\left|V_{i}\right|>2$, and joining $w_{i}$ with every vertex in $V_{i}$. In this paper, we study the distance properties of degree splitting graphs. We classify the graphs into three families based on the variation of diameter of a graph from that of its degree splitting graph and obtain few results on them.
\end{abstract}

Keywords: Degree splitting graphs, diameter, radius, self centered graphs.

\section{INTRODUCTION}

The graphs taken under consideration in this paper are finite, simple and undirected. For notations and terminology, we refer [7]. The degree of a vertex $v$ is denoted by $d(v)$. A graph $G$ is said to be $r-$ regular, if every vertex of $G$ has degree $r$.

Let $G(V, E)$ be any graph. For any vertex $v \in$ $V(G)$, the open neighbourhood $N(v)$ of $v$ is the set of all vertices adjacent to $v$. That is, $N(v)=\{u \in V(G)$ / $u v \in E(G)\}$. The closed neighbourhood of $v$ is defined by $N[v]=N(v) \cup\{v\}$. Let $V_{i}=\{v \in V(G) /$ $d(v)=i\}$. Note that in an $r$ regular graph $G, V_{r}=$ $V(G)$ and $V_{i}=\phi$ for any $i \neq r$. A vertex $v$ in $\mathrm{G}$ is called a distinguished vertex of $G$ if $v$ is the only vertex in $G$ with degree $d(v)$.

A full vertex is a vertex $v$ in $G$ which is adjacent to all other vertices in $G$, that is $d(v)=$ $|V(G)|$ - 1.A vertex of degree one is called a pendant vertex. A vertex $v$ is said to be a $k$ - regular adjacency vertex (or simply a $k-R A$ vertex) if $d(u)=$ $k$ for all $u \in N(v)$. A vertex is called an $R A$ vertex if it is a $k-R A$ vertex for some $k \geq 1$. A graph $G$ in which every vertex is an $R A$ vertex is said to be an $R A$ graph. For example, any regular graph and the complete bipartite graph $K_{m, n}, m \neq n$ are $R A$ graphs.

Let $G_{l}$ and $G_{2}$ be any two graphs. The graph $G_{1} \circ G_{2}$ obtained from one copy of $G_{I}$ and $\left|V\left(G_{1}\right)\right|$ copies of $G_{2}$ by joining each vertex in the $\mathrm{i}^{\text {th }}$ copy of $G_{2}$ to the $\mathrm{i}^{\text {th }}$ vertex of $G_{1}$ is called the corona of $G_{l}$ and $G_{2}$. The join of two graphs $G_{1}$ and $G_{2}$ is denoted by $G_{1} \vee G_{2}$.

For any two vertices $u$ and $v$, let $d(u, v)$ denote the distance, that is, the length of a shortest path between $u$ and $v$. The eccentricity, $e(u)$ of a vertex $u$ is the distance of a farthest vertex from $u$. Radius, $\operatorname{rad}(G)$ of a graph $G$ is the minimum eccentricity of the graph whereas diameter $\operatorname{diam}(G)$ is its maximum eccentricity. A graph with $\operatorname{rad}(G)=$ $\operatorname{diam}(G)$ is called a self centered graph. For basic definitions and elementary results on distance in graphs one can refer [8]. Splitting graph of a graph is a concept introduced by Sampath Kumar and Walikar [10]. This concept resembles the method of taking clone of every vertex in a graph. The definition of splitting graph is given as follows: The graph $S(G)$ obtained from $G$, by taking a new vertex $v^{\prime}$ for every vertex $v \in V$ and joining $v^{\prime}$ to all vertices of $G$ adjacent to $v$, is called a splitting graph of $G$. As an illustration, a graph $G$ with its splitting graph $S(G)$ is shown in Figure 1.

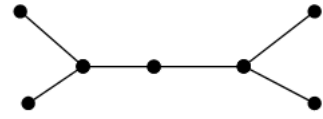

$G$

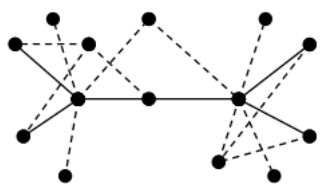

$S(G)$

Figure 1 
The properties of splitting graphs have been studied and the necessary and sufficient conditions for a graph to be a splitting graph have been discussed in [10]. Distance properties of splitting graphs have been studied in [5]. On a similar line, recently instead of adding a clone for every vertex, a method of adding complement to every vertex has been introduced and studied for its properties [1].

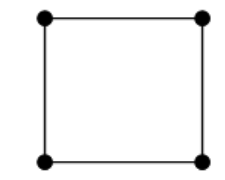

$G$
Let $G$ be a graph with vertex set $\left\{v_{l}, v_{2}, \ldots\right.$, $\left.v_{n}\right\}$. The cosplitting graph $C S(G)$ is the graph obtained from $G$, by adding a new vertex $w_{i}$ for each vertex $v_{i}$ and joining $w_{i}$ to all vertices which are not adjacent to $v_{i}$ in $G$.

For instance, a graph $G$ and its cosplitting graph $C S(G)$ are shown in Figure 2.

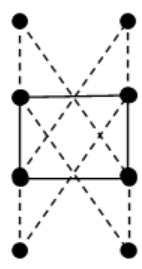

$\operatorname{CS}(G)$

Figure 2

In [1], a necessary and sufficient condition for a graph to be a cosplitting graph has been given. And the graphs for which the splitting graph and the cosplitting graph are isomorphic have been characterised. Further results on cosplitting graphs can be referred in [1] and [5].

Recently on this sequence, the concept of $\gamma$ splitting graphs has been introduced and studied by Selvam Avadayappan et al. [4]. In a graph $G$, a subset $S$ of $V$ is called a dominating set of $G$ if every vertex in $\mathrm{V}-\mathrm{S}$ is adjacent to at least one vertex in $S$. The domination number of a graph denoted by $\gamma$ is the

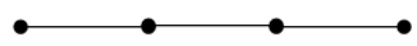

$P_{4}$

One such related concept namely $\beta$ splitting graphs has been introduced and studied by Selvam Avadayappan et al. [6]. Let $S_{1}, S_{2}, \ldots, S_{\rho}$ be the maximum independent sets of $G$, where $\rho$ is the number of maximum independent sets in $G$. The $\beta$ - splitting graph $S_{\beta}(G)$ of a graph $\mathrm{G}$ is a graph obtained from $\mathrm{G}$ by adding new vertices number of vertices in a minimum dominating set and any minimum dominating set is referred as $\gamma-$ set.

Let $G$ be a graph and let $\eta$ be the number of $\gamma$ - sets in $G$. Let $S_{1}, S_{2}, \ldots, S_{\eta}$ be the $\gamma$ - sets in $G$. The $\gamma$ - splitting graph, $S_{\gamma}(G)$, of a graph $G$ is the graph obtained from $G$ by adding new vertices $w_{l}$, $w_{2}, \ldots, w_{\eta}$ and joining to each vertex in $\mathrm{S}_{\mathrm{i}}$ for $1 \leq i \leq \eta$. For example, the graph $P_{4}$ and its $\gamma-$ splitting graph $S_{\gamma}\left(P_{4}\right)$ are shown in Figure 3.

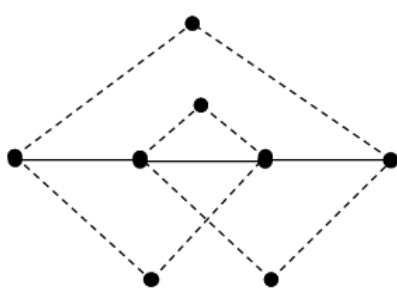

$S_{\gamma}\left(P_{4}\right)$

Figure 3

$w_{1}, w_{2}, \ldots, w_{\rho}$ such that each $w_{i}$ is adjacent to each vertex in $S_{i}$, for $1 \leq i \leq \rho$. For example, a graph $\mathrm{G}$ and its $\beta$-splitting $\operatorname{graph} S_{\beta}(G)$ are shown in Figure 4. 


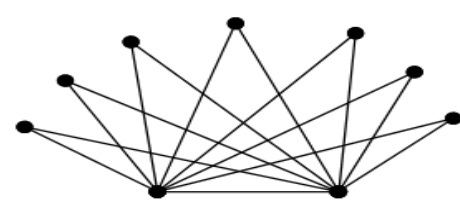

$\boldsymbol{G}$

One another type of splitting graph has started its account with the introduction of degree splitting graph by Ponraj and Somasundaram [9]. The degree splitting graph $D S(G)$ of a graph $G$ can be defined as follows: For a graph $G(V, E)$, the degree

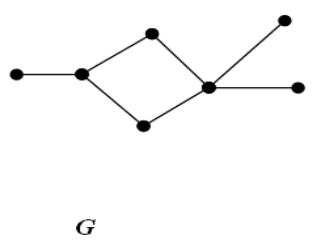

It is easy to note that, if $G$ is regular, then $D S(G)$ is nothing but $G \vee K_{l}$ and trivial graph is the only graph for which the degree splitting graph is isomorphic to itself. Every graph is an induced subgraph of its own degree splitting graph. Note that any newly added vertex $w$ in $D S(G)$ is an $R A$ vertex. As any graph $G$ contains at least two vertices of same degree, any degree splitting graph $D S(G)$ contains at least one $R A$ vertex.

For further results on degree splitting graphs, one can refer [2] and [3].

The necessary conditions for a graph to be a degree splitting graph for some graph has been discussed in [3] and an algorithm to check whether the given graph is a degree splitting graph or not has also been developed. In addition for any $n \geq 2$, the existence of $n$ non isomorphic graphs, $G_{1}, G_{2}, \ldots, G_{n}$ having isomorphic degree splitting graph has been proved in [3]. Some necessary conditions for the existence of non-isomorphic graphs with isomorphic degree splitting graph have been given in [3].

Thinking of the distance properties of degree splitting graphs, we normally expect the diameter of a degree splitting graph of a graph $G$ to be less than that of $\mathrm{G}$, as the newly added vertices usually reduce the distance between the non adjacent vertices of same degree. But to our surprise there exist graphs with diameter less than that of its degree splitting graph. Based on this varied behaviour, we classify the graphs into three families and discuss their properties and structure in this paper.

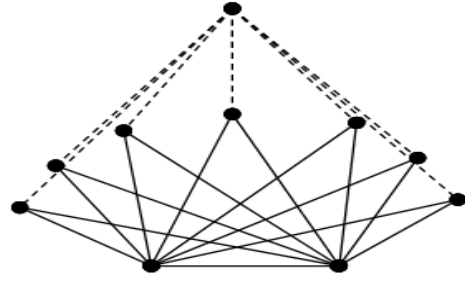

$$
S_{\beta}(G)
$$

\section{Figure 4}

splitting graph $\operatorname{DS}(G)$ is obtained from $G$, by adding a new vertex $w_{i}$ for each partition $V_{i}$ that contains at least two vertices and joining $w_{i}$ to each vertex of $V_{i}$. For example, a graph $G$ and its degree splitting graph $D S(G)$ are shown in Figure 5.

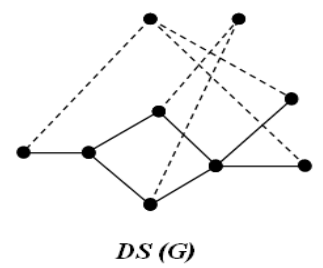

Figure 5

\section{DISTANCE IN DEGREE SPLITTING GRAPHS}

The following facts can be easily verified while finding the diameter of degree splitting graphs of some basic graphs.

Fact $1 \operatorname{diam}(D S(G))=1$ if and only if $G \cong K_{n}$.

Fact $2 \operatorname{diam}\left(D S\left(P_{n}\right)\right)=\left\{\begin{array}{l}n-1 \text { if } n=2,3,4 \\ 3 \quad \text { if } n=5 \text { or } 6 \\ 4 \quad \text { if } n \geq 7\end{array}\right.$

Fact $3 \operatorname{diam}\left(D S\left(C_{n}\right)\right)=\operatorname{diam}\left(D S\left(W_{n}\right)\right)=$ $\operatorname{diam}\left(D S\left(K_{1, n-1}\right)\right)=2$, for all $n \geq 3$.

Fact $4 \operatorname{diam}\left(D S\left(K_{m, n}\right)\right)=3$ if $m \neq n$, 2 otherwise.

Proposition 2.1 For any regular graph $G$, $\operatorname{diam}(D S(G)) \leq 2$.

Proof Let $G$ be any regular graph. Then $D S(G)=G$ $\vee K_{l}$. Therefore $\operatorname{diam}(D S(G)) \leq 2$.

Corollary $2.2 \operatorname{diam}(D S(G))=2$, for any noncomplete regular graph $G$.

Theorem 2.3 Let $G$ be a graph with $\left|V_{i}\right| \geq 2$ for at least two positive integers $i$. Then $\operatorname{diam}(D S(G)) \geq 3$.

Proof Let $G$ be any graph in which $\left|V_{i}\right| \geq 2$ and $\left|V_{j}\right| \geq$ 2 for some $i$ and $j, i \neq j$. Then, $D S(G)$ contains at least two newly added vertices, say $w_{i}$ and $w_{j}$ corresponding to $V_{i}$ and $V_{j}$ respectively.

Since $i \neq j, N\left(w_{i}\right) \cap N\left(w_{j}\right)=\phi$ in $D S(G)$. Hence $d\left(w_{i}, w_{j}\right) \geq 3$. This forces that $\operatorname{diam}(D S(G)) \geq 3$.

Theorem 2.4 Let $G$ be a graph with exactly one full vertex. Then $2 \leq \operatorname{diam}(D S(G)) \leq 4$.

Proof Let $G$ be any graph of order $n$ with $\Delta(G)=n-$ 1 and let $v$ be the full vertex in $G$. While considering 
degree splitting graph $D S(G)$ of $G$, the following two cases arises.

Case $1 D S(G)$ contains exactly one newly added vertex, say $w$

Since $v$ is a full vertex in $G, e(u)=2$ for any vertex $u$ other than $v$ in $G$. Since $\left|V_{n-1}\right|=1, v \notin N(w)$, for any newly added vertex $w$. Also it is obvious that $N(v) \cap N(w) \neq \phi$. Let $x \in N(v) \cap N(w)$. Henced $(v, w)$ $=2$ and so $e(v)=2$. For any other vertex $u, u v x w$ is a $(u, w)$ - path of length three. Hence $e(u) \leq 3$ for every vertex other than $v$ in $D S(G)$. This makes us conclude that $2 \leq \operatorname{diam}(D S(G)) \leq 3$.

Case $2 D S(G)$ contains more than one newly added vertices.

As in Case 1, we can easily note that $e(v)=$ 2 in $D S(G)$. Let $w_{1}$ and $w_{2}$ be any two newly added vertices in $D S(G)$. Then since $w_{1}$ and $w_{2}$ are $R A$ vertices, $N\left(w_{1}\right) \cap N\left(w_{2}\right)=\phi$. Now $d\left(w_{1}, w_{2}\right) \leq 4$ as $w_{1} v_{1} v v_{2} w_{2}$ is a $\left(w_{1}, w_{2}\right)$ - path of length four in $D S(G)$, where $v_{1} \in N\left(w_{1}\right), v_{2} \in N\left(w_{2}\right)$. For any vertex $u$ in $G$ other than $v, d\left(u, w_{1}\right) \leq 3$ and $d\left(u, w_{2}\right) \leq 3$. This forces that $e(u) \leq 3$. Also by Theorem 2.3, we have $\operatorname{diam}(D S(G)) \geq 3$. Therefore $3 \leq \operatorname{diam}(D S(G)) \leq 4$.
Combining both the cases, we note that $2 \leq$ $\operatorname{diam}(D S(G)) \leq 4$.

Note that the above said inequalities obtained in the above theorem are strict which have been proved in following theorems.

Proposition 2.5 For any integer $n \geq 3$, there exists a graph $G$ of order $n$ and maximum degree $n-1$ such that $\operatorname{diam}(D S(G))=2$.

Proof Consider a graph $G=K_{1, n-1}$. We know that $D S(G)=K_{2, n-1}$. Here $\operatorname{diam}(D S(G))=2$.

Theorem 2.6 For any integer $n \geq 5$, there exists a graph $\mathrm{G}$ of order $\mathrm{n}$ and maximum degree $\mathrm{n}-1$ such that $\operatorname{diam}(\mathrm{DS}(\mathrm{G}))=4$.

Proof Construct a graph $\mathrm{G}$ with $\mathrm{V}(\mathrm{G})=\left\{\mathrm{v}, \mathrm{v}_{1}, \mathrm{v}_{2}, \ldots\right.$, $\left.\mathrm{v}_{\mathrm{n}-1}\right\}$ and $\mathrm{E}(\mathrm{G})=\left\{\mathrm{vv}_{\mathrm{i}} / 1 \leq \mathrm{i} \leq \mathrm{n}-1\right\} \cup\left\{\mathrm{v}_{\mathrm{i}} \mathrm{v}_{\mathrm{j}} / 1 \leq \mathrm{i}, \mathrm{j}\right.$ $\leq \mathrm{n}-3, \mathrm{i} \neq \mathrm{j}\}$. $\mathrm{G}$ is nothing but a graph obtained by attaching two pendant vertices at any vertex of $K_{n-2}$. One can easily check that $\mathrm{G}$ is a graph of order $\mathrm{n}$ with a full vertex such that $\operatorname{diam}(\mathrm{DS}(\mathrm{G}))=4$. Hence the theorem.

The case when $n=7$ is illustrated in Figure 6.

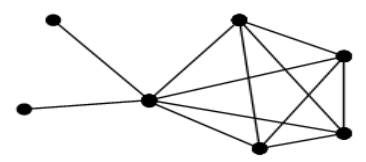

$\boldsymbol{G}$

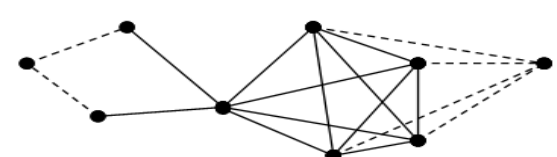

$\operatorname{DS}(G)$

Figure 6

It is important to note here that when we attach a single pendant vertex at any vertex of a complete graph, we get a graph $G$ of order $n$ and maximum degree $n-1$ such that $\operatorname{diam}(D S(G))=3$.

\section{CLASSIFICATION OF GRAPHS INTO DIFFERENT FAMILIES}

In any degree splitting graph, vertices of same degree are at a distance at most two. Hence we normally expect diameter of the degree splitting graph $D S(G)$ of a graph to be less than that of the graph $G$ itself. That is, we may think that the diameter of $D S(G)$ is always less than or equal to that of $G$.

But while analysing distance properties of degree splitting graphs we find that diameter property is totally independent of degree splitting. In fact, there are graphs whose diameter is less than or equal to the diameter of its degree splitting graph. This variation is studied in detail in this section.

Let us first classify graphs into three various groups and discuss their properties.

A graph $G$ is said to be a $D S^{+}$- graph if $\operatorname{diam}(D S(G))>\operatorname{diam}(G)$ while it is said to be a $D S^{-}$- graph if $\operatorname{diam}(D S(G))<\operatorname{diam}(G)$ and $\mathrm{G}$ is called $D S^{*}$ - $\operatorname{graph}$ if $\operatorname{diam}(D S(G))=\operatorname{diam}(G)$.

It is easy to verify that $K_{n}$ and $W_{n}$ are $D S^{*}$ graphs and $P_{n}$ and $C_{n}(n \geq 3)$ are $D S^{-}$- graphs. But the complete bipartite graphs $K_{m, n}, m \neq n$, is a $D S^{+}$graph.

Theorem 3.1 For any $n \geq 4$, there exists a self centered $D S^{*}$ - graph $G_{n}$ such that $D S\left(G_{n}\right)$ is also self centered.

Proof Construct a graph $G_{n}$ with vertex set $V\left(G_{n}\right)=$ $\left\{u, v_{l}, v_{2}, \ldots \ldots, v_{n-l}\right\}$ and edge set $E\left(G_{n}\right)=\left\{u v_{l}, u v_{n-1}, v_{i}\right.$ $\left.v_{j}, 2 \leq i, j \leq n-2, v_{r} v_{s}, r=1, n-1,2<s<n-2\right\}$. It is clear that every vertex in $G_{n}$ has eccentricity two and hence $G_{n}$ is a self centered graph of radius two.

Note that $G_{n}$ is nothing but a graph obtained from $K_{n-1}$ by subdividing only one edge. In $G_{n}, u$ is a vertex of degree two and all other vertices are of degree $n-2$. Hence $D S\left(G_{n}\right)$ contains one newly added vertex adjacent to each $v_{i}, 1 \leq i \leq n-1$.

One can easily check that $D S\left(G_{n}\right)$ contains no full vertex and is also of diameter two and hence a self centered graph. And also by definition, since $\operatorname{diam}\left(G_{n}\right)=\operatorname{diam}\left(D S\left(G_{n}\right)\right), G_{n}$ is a self centered DS* graph.' 
For example when $n=7$, the constructed self centered DS* - graph $G_{7}$ is shown in Figure7.

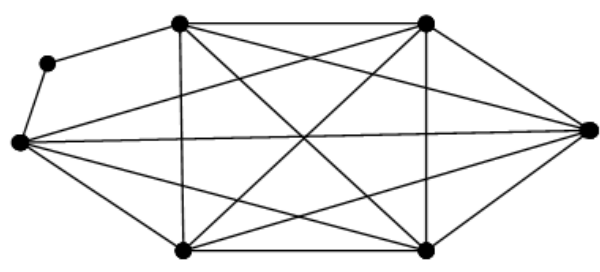

$G_{7}$

Figure 7

Theorem 3.2 For any $d \geq 3$, there exists a $D S^{*}$ - graph of diameter $d$.

Proof Let $d \geq 3$ be any given integer. When $d$ is of the form $3 m-1$, construct a graph $G$ with vertex set $V(G)=\left\{v_{i j}, 1 \leq i \leq m, 1 \leq j \leq i+3, u_{i}, 1 \leq i \leq 2 m\right\}$ and edge set $E(G)=\left\{v_{i j} v_{i k}, \quad l \leq i \leq m, 1 \leq j, k \leq i+3, j \neq k\right.$; $v_{i j} u_{k}, 1 \leq i \leq m, 1 \leq j \leq i+3,2 i-1 \leq k \leq 2 i ; u_{2 i} u_{2 i+1}, 1 \leq i$ $\leq m-1\}$.

In the constructed graph $G$, the diametral vertices are $u_{1}$ and $u_{2 m}$ which are at a distance $3 m-1$. Also in $G$, for every $i, 1 \leq i \leq m, u_{2 i-1,}, u_{2 i}$ and $v_{i j}$ 's, $1 \leq j$ $\leq i+3$, are all of degree $i+3$. Hence $\mathrm{m}$ new vertices namely $w_{1}, w_{2}, \ldots, w_{m}$ are newly added in $D S(G)$.

It is easy to observe that $w_{i}$ is an isomorphic image of any $v_{i j}$, where $1 \leq i \leq m$. Hence again in $D S(G), u_{1}$ and $u_{2 m}$ are diametral vertices at a distance $3 m-1$. Therefore $G$ is a $D S^{*}-$ graph of diameter $d$.

If $d \equiv 0(\bmod 3)$, construct a graph $H$ by adding a pendant vertex $u_{2 m+1}$ to $u_{2 m}$ in $G$. Then $H$ is the required $D S^{*}$ - graph. When $d=3 m+1$, attach a pendant vertex to $u_{2 m+1}$ in $H$. The resultant graph is the required $D S^{*}$ - graph.

For example, a $D S^{*}$ - graph of diameter 5 is shown in Figure 8.

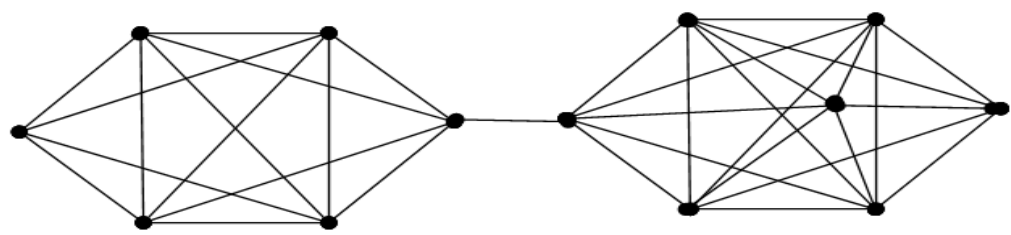

Figure $s$

Theorem 3.3 Any regular graph $G$ is a $D S^{*}-$ graph if and only if it is complete or self centered with radius two.

Proof Let $G$ be any regular graph. Then $D S(G)=$ $G \vee K_{l}$. Then the diameter of $D S(G)$ is at most two. If $\operatorname{diam}(D S(G))=1$, then by Fact 2.1, G is a complete graph.

If $\operatorname{diam}(D S(G))=2$, then $\operatorname{rad}(G) \leq 2$. Since $G$ is regular, if $\operatorname{rad}(G)=1$, then $G$ is complete which is a contradiction to the fact that $\operatorname{diam}(G)=2$. Thus we conclude that $G$ is a self centered graph with radius two.

Theorem 3.4 Let $G$ be any non - complete graph with a full vertex. Then $G$ is a $D S^{*}$ - graph if and only if $G$ is a graph with vertex set $V(G)=$ $\cup_{i=\delta}^{\Delta} V_{i}$ satisfying the following conditions: (i) $\left|V_{j}\right| \geq$ 2 for some unique $j, \delta \leq j \leq \Delta$ and (ii) $V(G) \backslash V_{j} \subseteq N\left(V_{j}\right)$.

Proof Let $G$ be any non complete graph with a full vertex. Therefore $\operatorname{diam}(G)=2$. Consider the vertex set partition of $G, V(G)=\bigcup_{i=\delta}^{\Delta} V_{i}$ such that $V_{i}$ contains vertices of degree $i$. Suppose that $G$ is a $D S^{*}$ - graph. Then $\operatorname{diam}(D S(G))=2$.

Suppose if possible assume that at least two vertices say, $u$ and $v$ are newly added in $D S(G)$. Then as in the proof of Theorem 2.3, we have $d(u, v) \geq 3$, which is a contradiction. Therefore $\left|V_{j}\right| \geq 2$ for some unique $j, \delta \leq j \leq \Delta$. Hence (i) holds.

Let $w_{j}$ be the corresponding unique newly added vertex in $D S(G)$ corresponding to $\mathrm{v}_{\mathrm{j}}$.

Now if possible let $w$ be a vertex of $G$ with degree not equal to $j$ and not adjacent to any vertex of degree $j$. That is, let $w \in V(G) \backslash V_{j}$ and $w \notin$ $N\left(V_{j}\right)$. Then $d\left(w, w_{j}\right)>2$ in $G$ which is a contradiction to the fact that $G$ is a $D S^{*}(G)$ graph of diameter 2 . Hence (ii) follows.

Conversely, Let $G$ be any non-complete graphwhich satisfies the given conditions. Since $G$ contains a full vertex, $\operatorname{diam}(G)=2$. Also by (i) only one vertex, say $w_{j}$, is newly added to $D S(G)$. Again by (ii), every vertex of degree not equal to $j$ is a neighbour of at least one vertex of degree $j$. 
Therefore $d\left(w_{j}, w\right) \leq 2$, for every vertex $w \in$ $D S(G)$. This forces that $\operatorname{diam}(D S(G)) \leq 2$. Since $G$ is not complete, we conclude that $\operatorname{diam}(D S(G))=2$. Hence $G$ is a $D S^{*}$ - graph.

Corollary 3.5 If $G$ is a graph with at least one full vertex, then $G$ is a $D S^{*}$ - graph or $D S^{+}$- graph.

Proof Suppose $G$ is complete. Then $G$ is a $D S^{*}$ graph. If $G$ is not complete, then it is a $D S^{*}$ graphwhen it satisfies the conditions given in Theorem 3.4.

If (i) fails, that is, if $\left|V_{j}\right|$ and $\left|V_{k}\right| \geq 2$ for some $j \neq k$ where $\delta \leq j, k \leq \Delta$, then $\operatorname{diam}(D S(G))>2$. If $V_{j} \cap N\left(V_{k}\right) \neq \phi$, then $\operatorname{diam}(D S(G))=3$. Otherwise $\operatorname{diam}(D S(G))=4$. Also if (i) holds and (ii) fails, then $\operatorname{diam}(D S(G))=3$.

In all the cases, $G$ is a $D S^{+}$- graph.

Corollary 3.6 A $D S^{*}$ - graph $G$ contains more than one full vertex if and only if $G \cong K_{n}$.

Proof Let $G$ be a $D S^{*}$ - graph of order $n$ with at least two full vertices. That is, $\left|V_{n-1}\right| \geq 2$ in $G$. Then by Theorem 3.4, $\left|V_{i}\right| \leq 1$, for every $i, \delta \leq i \leq n-2$.

Let $\mathrm{H}$ be the graph obtained from $\mathrm{G}$ by deleting all full vertices of $\mathrm{G}$. Then in $\mathrm{H}$, no two vertices are of same degree. But it is well known that no such graph $\mathrm{H}$ exists. Hence $G \cong K_{n}$. The converse is obvious.

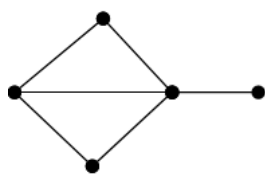

$G$

Figure 9

Theorem 3.9 Any regular graph other than complete or self centered graph of radius two is a $D S^{-}$- graph.

Proof Let $G$ be a regular graph which is neither a complete graph nor a self centered graph of radius two. Then $G$ contains at least two vertices $u$ and $v$ such that $d(u, v) \geq 3$. Hence $\operatorname{diam}(G) \geq 3$. Now $D S(G)$ $=G \vee K_{l}$. Then distance between any two vertices in $D S(G)$ is at most 2. Hence $\operatorname{diam}(D S(G))=2$ which implies $G$ is a $D S^{-}$- graph.

Theorem 3.10 For any $n \geq 6$, there exists a self centered $D S^{-}$- graph of order $n$.

Proof Consider the graph $C_{n}, n \geq 6$. We know that $\operatorname{diam}\left(C_{n}\right)=\left\lfloor\frac{n}{2}\right\rfloor$. Also for every vertex $u$ in $C_{n}, e(u)=$ $\left\lfloor\frac{n}{2}\right\rfloor$. Hence $C_{n}$ is a self centered graph of order $n$. Now $D S\left(C_{n}\right)=K_{l} \vee C_{n}$. And so $\operatorname{diam}\left(\operatorname{DS}\left(C_{n}\right)\right)=2$. Therefore $C_{n}$ is a $D S^{-}-$graph and hence the theorem. -

Corollary 3.11 For any $n \geq 3$, there exists a $D S^{-}$graph with diameter equal to $n$.
Corollary 3.7 For any non-completegraph $G, G \vee K_{n}$, where $n \geq 2$ is a $D S^{+}$- graph.

ProofThe result follows immediately from Corollary 3.5 and Corollary 3.6.

Theorem 3.8 Every graph is an induced subgraph of a $D S^{*}$ - graph.

Proof If $G$ itself is a $D S^{*}$ - graph, then there is nothing to prove. Assume that $G$ is not a $D S^{*}$ - graph. Then $G$ is not complete. First we construct a regular graph $\operatorname{Reg}(G)$ which contains $G$ as an induced subgraph.

If $G$ is a regular graph, then take $\operatorname{Reg}(G)=$ $G$. If not, let $V(G)=\left\{v_{1}, v_{2}, \ldots, v_{n}\right\}$ be the vetex set of G. Now construct the graph $\operatorname{Reg}(G)$ with vertex set $V(\operatorname{Reg}(G))=\left\{u_{1}, u_{2}, \ldots, u_{n}, w_{1}, w_{2}, \ldots, w_{n}\right\}$ and edge set $E(\operatorname{Reg}(G))=\left\{u_{i} u_{j}, w_{i} w_{j} / v_{i} v_{j} \in E(G), 1 \leq i, j \leq n\right\} \cup$ $\left\{u_{i} w_{j} / v_{i} v_{j} \notin E(G)\right.$ and $\left.1 \leq i, j \leq n\right\}$. From our construction, $\operatorname{Reg}(G)$ is a $\mathrm{n}$ - regular graph on $2 \mathrm{n}$ vertices.

Take $H=K_{l} \vee \operatorname{Reg}(G)$. Clearly $H$ contains $G$ as an induced subgraph and $\operatorname{diam}(H)=2$. Also $D S(H)=K_{I} \vee H$. Therefore $\operatorname{diam}(D S(H))=2$. Hence $H$ is the required $D S^{*}$ - graph which contains $G$ as an induced subgraph.

For example, for the graph $G, D S^{*}$ - graph $H$ which contains $G$ as induced subgraph is shown in Figure 9.

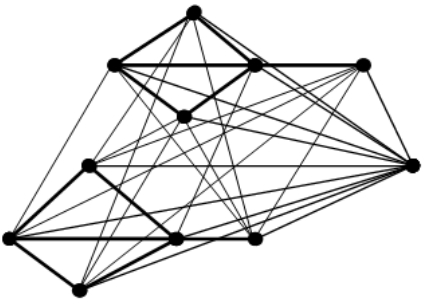

$H$

Proof The graph $C_{2 n}$ is an example for the existence of $D S^{-}$- graph of diameter $n$.

Corollary 3.12 For any $m \geq 1$, there exists a $D S^{-}$$\operatorname{graph}$ such that $\operatorname{diam}(D S(G))+m=\operatorname{diam}(G)$.

Proof Consider $G=C_{2 m+4}$. It is easy to note that $\operatorname{diam}(G)=m+2$. Obviously $D S(G)$ is a graph with full vertex and hence $\operatorname{diam}(D S(G))=2$ which proves the corollary.

Theorem 3.13 Every graph of diameter at least 5 is an induced subgraph of a $D S^{-}$- graph.

Proof Let $G$ be any graph of diameter at least 5 . Consider $H=G \circ K_{1}$. Now $\operatorname{diam}(H)=\operatorname{diam}(G)+2$. Hence $\operatorname{diam}(H) \geq 7$. Clearly $G$ is an induced subgraph of $H$. In $H$, every vertex other than pendant vertex is a neighbour of a pendant vertex. Also $D S(H)$ contains a newly vertex adjacent to all the pendant vertices of $H$. Therefore distance between any two vertices of $H$ in $D S(H)$ is always less than or equal to 4 .

In addition, it can be easily verified that the distance between any two newly added vertices in 
$D S(H)$ is at most 6 . Then $\operatorname{diam}(D S(H)) \leq 6$ implying that $H$ is a $D S^{-}-$graph
For example, a graph $G$ and the corresponding graph $D S^{-}$- graph $H$ constructed with its degree splitting graph are shown in Figure 10.

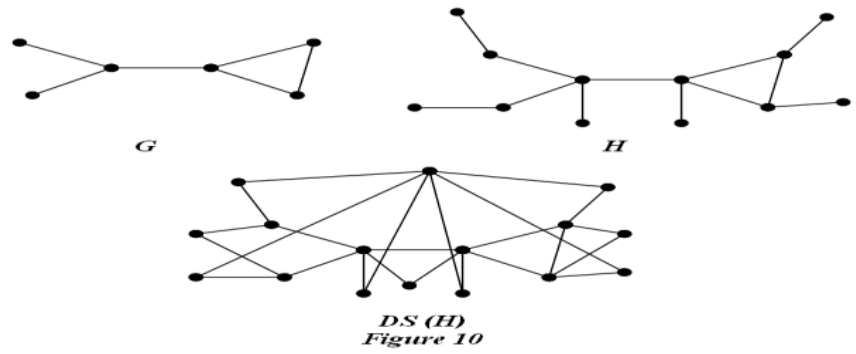

Note that for any graph $G$ of diameter at least 3, $\operatorname{Reg}(G)$ is a $D S^{-}$- graph which contains $G$ as an induced subgraph.

Theorem 3.14 Let $G$ be a $D S^{+}$- graph. Then the diameter of $G$ differs from that of its degree splitting graph by at most 2 . That is, $\operatorname{diam}(D S(G))=\operatorname{diam}(G)$ $+1 \operatorname{ordiam}(G)+2$.

Proof On contrary assume that there exists a $D S^{+}$graph $G$ with diameter $d$ such that $\operatorname{diam}(D S(G))=t \geq$ $d+3$. Let $P=v_{0} v_{1} v_{2} \ldots v_{t}$ be a diametral path of length t in $D S(G)$. Clearly $P$ contains some newly added vertices, since $t>d$. One can easily note that no newly added vertex can be an internal vertex of $P$. This forces that $v_{0}$ or $v_{t}$ or both are the newly added vertices in $P$.

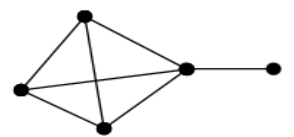

$G$

Now let us consider a path $Q=P-\left\{v_{0}, v_{t}\right\}$. Then length of $Q$ is $t-2 \geq d+1$. Also $Q$ contains only vertices of $G$. This means that $\operatorname{diam}(G) \geq d+1$ which is a contradiction. Hence $\operatorname{diam}(D S(G))=\operatorname{diam}(G)+$ 1 or $\operatorname{diam}(G)+2$.

Theorem 3.15 For any $n \geq 4$, there exists a $D S^{+}$$\operatorname{graph} G$ of order $n$ such that $\operatorname{diam}(D S(G))=\operatorname{diam}(G)$ +1 .

Proof Consider $G=K_{1} \cup\left(K_{n-3} \cup K_{1}\right)$, a graph of order $n$. We know that diameter of $G$ is 2 . $D S(G)$ contains one newly added vertex forcing $\operatorname{diam}(D S(G))$ to be 3 . Hence the theorem follows.

For $n=5$, the corresponding graph $G$ and $D S(G)$ are shown in Figure 11.

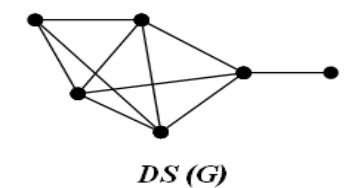

Figure 11

Theorem 3.16 For any $n \geq 5$, there exists a $D S^{+}$graph $G$ of order $n$ such that $\operatorname{diam}(D S(G))=\operatorname{diam}(G)$ +2 .

Proof For any given $n \geq 5$, consider a graph $G=$ $K_{1} \cup\left(K_{n-2} \cup K_{2}^{c}\right)$ of order $n . D S(G)$ is a graph of diameter four which proves the theorem.

The graph $G$ constructed for $n=6$ with its $D S(G)$ is given in Figure 12 .

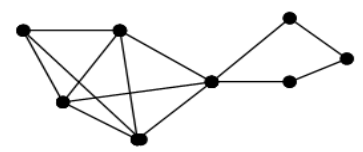

$D S(G)$

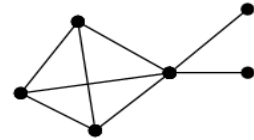

$G$

Figure 12

Theorem 3.17 Every graph $G$ of order at least three is an induced subgraph of a $D S^{+}$- graph.

Proof If the given graph $G$ itself is $D S^{+}$- graph, then there is nothing to prove. If not, for $G$ with vertex set
$V(G)=\left\{v_{l}, v_{2}, \ldots, v_{n}\right\}$, construct the graph $\operatorname{Reg}(G)$ as in the proof of Theorem 3.8.It is noteworthy here that if $G$ is a graph of order $n, \operatorname{Reg}(G)$ is an $n-$ regular graph on $2 n$ vertices. Also it is given that $n \geq 3$. 
Now fix an arbitrary vertex $\mathrm{u}$ in $\operatorname{Reg}(G)$. Delete two arbitrary edges incident with $u$ in $\operatorname{Reg}(G)$, say $u v$ and $u w$. Let the resultant graph be denoted by $H_{l}$. Now construct a graph $H$ from $K_{l} \vee H_{l}$ by adding two pendant vertices $u_{1}$ and $u_{2}$ at $u$, a new pendant vertex $x$ at $v$, a new pendant vertex $y$ at $w$ and a new edge $x y$. Clearly $H$ contains exactly two vertices of degree one and two vertices of degree two. Now $\operatorname{diam}(H)=4$ with $u$ and $x$ as diametral vertices.
Also $D S(H)$ contains three newly added vertices. And the newly added vertices corresponding to vertices of degree one and two are the terminal vertices of a diametral path of length. This implies that $H$ is a $D S^{+}$- graph which contains $G$ as an induced subgraph. Hence the theorem follows.

For example, a graph $G$ and the corresponding graph $D S^{+}$- graph $H$ constructed with its degree splitting graph are shown in Figure 13.

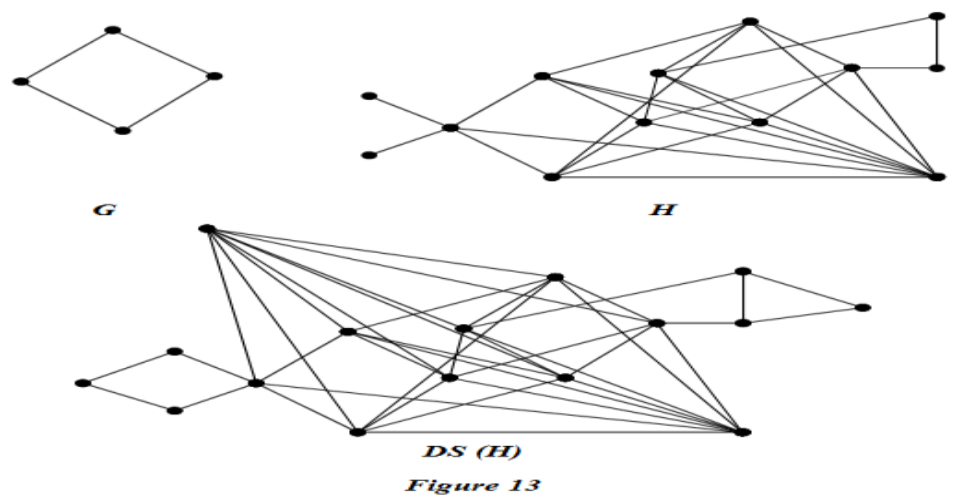

\section{REFERENCES}

[1] Selvam Avadayappan and M. Bhuvaneshwari, Cosplitting graph and coregular graph, International Journal of Mathematics and Soft Computing, Vol.5, No.1,(2015), 57 -64.

[2] Selvam Avadayappan and M. Bhuvaneshwari, Some results on Degree splitting graphs, International Journal of Advanced and Innovative Research, Vol . 5, Issue 3, March 2016.

[3] Selvam Avadayappan and M. Bhuvaneshwari, Isomorphic Degree splitting graphs (Preprint).

[4] Selvam Avadayappan, M. Bhuvaneshwari and R. Iswarya, $\gamma$-splitting graphs, International Journal for Research in Applied Science \& Engineering Technology, Vol. 4, Issue III, March 2016, $670-680$.

[5] Selvam Avadayappan, M. Bhuvaneshwari and Rajeev Gandhi, Distance in splitting graphs, International Journal of Engineering
Sciences and Management Research, 2(12), 50 -54, December 2015.

[6] Selvam Avadayappan, M.Bhuvaneshwari and B.Vijaya Lakshmi, $\beta$-Splitting graphs, International Journal of Scientific Review and Research in Engineering and Technology, Vol. 1(3), Mar. - Apr. 2016, 84 $-101$.

[7] R.Balakrishnan and K. Ranganathan, A Text Book of graph Theory, Springer-Verlag, New York, Inc(1999).

[8] F. Buckley and F. Harary, Distance in Graphs, Addison-Wesley Reading, 1990.

[9] R.Ponraj and S.Somasundaram, On the degree splitting graph of a graph, NATL. ACAD. SCI. LETT., Vol-27, No.7 \& 8, pp. 275 - 278, 2004.

[10] Sampath Kumar.E, Walikar.H.B, On theSplitting graph of a graph,(1980), J.Karnatak Univ. Sci 25: 13.

\footnotetext{
International Journal of Engineering Research and Applications (IJERA) is UGC approved Journal with S1. No. 4525, Journal no. 47088. Indexed in Cross Ref, Index Copernicus (ICV 80.82), NASA, Ads, Researcher Id Thomson Reuters, DOAJ.
}

Selvam Avadayappan. "Distance in Degree Splitting Graphs." International Journal of Engineering Research and Applications (IJERA) 7.7 (2017): 14-21. 